\title{
CORRESPONDENCE
}

\section{Association of endemic coronaviruses with nasopharyngeal metabolome and microbiota among infants with severe bronchiolitis: a prospective multicenter study}

Pediatric Research (2021) 89:1594-1597; https://doi.org/10.1038/s41390020-01154-2

\begin{abstract}
BACKGROUND
Coronaviruses (CoV) are important human pathogens. During epidemics, CoVs contribute to up to $20 \%$ of community-acquired respiratory infections in adults. ${ }^{1}$ These viruses also play a major role in children-indeed, the endemic CoVs (CoV-NL63, 229E, HKU1, and OC43) are the third most common virus pathogen among infants hospitalized for bronchiolitis ("severe bronchiolitis"). ${ }^{2}$ These endemic CoVs continuously circulate in the human population and have annual peaks of activity in winter. $^{3}$ Furthermore, a novel CoV-severe acute respiratory syndrome coronavirus 2 (SARS-CoV-2) - is the cause of the coronavirus disease 2019 (COVID-19) pandemic. ${ }^{1}$

CoVs, a family within the Nidovirales order, are enveloped positive-stranded RNA viruses. ${ }^{4}$ In human CoVs, its subfamily is classified into two major genera: alpha (NL63 and 229E) and beta (HKU1, OC43, Middle East respiratory syndrome coronavirus [MERS-CoV], and SARS-CoV-1/2). ${ }^{4}$ The sparse literature on severe respiratory infections by endemic CoVs in children and adults is conflicting, with the clinical severity found to be differ by virus type in some studies ${ }^{5,6}$ but not others. ${ }^{7}$ Furthermore, the novel CoVs (MERS and SARS-CoV-1/2) are known to cause fatal acute respiratory infection in some populations. ${ }^{1}$ Despite the clinical and research importance, little is known about how different endemic CoVs interact with the host and bacterial community in the airway niche which contributes to downstream molecular pathways and pathobiology. Metabolome and microbiome profiling address this knowledge gap by comprehensively profiling functional small molecules and bacteria in the airway.

In this context, by using prospectively collected multicenter cohort data, we investigated the between-genus (alpha vs. beta) $\mathrm{CoV}$ differences in clinical characteristics, nasopharyngeal airway metabolome, and microbiome profiles among infants with severe bronchiolitis.
\end{abstract}

\section{METHODS}

This is an analysis of data from the 35th Multicenter Airway Research Collaboration (MARC-35) cohort study-a multicenter prospective cohort study of infants with severe bronchiolitis. The details of the study design, measurements, and analysis are described in the Online Supplement. Briefly, we enrolled 1016 infants (aged <1 year) hospitalized with bronchiolitis-according to the American Academy of Pediatrics guidelines ${ }^{8}$ in 17 sites across 14 US states (Table E1). Investigators collected nasopharyngeal airway samples within $24 \mathrm{~h}$ of hospitalization using a standardized protocol. $^{9}$ These samples underwent $(A)$ real-time PCR to test for respiratory viruses, ${ }^{2}$
(B) ultra-performance liquid chromatography-tandem mass spectrometry to profile the metabolome, ${ }^{10}$ and (C) 165 rRNA gene sequencing to profile the microbiota. ${ }^{11}$

In the current analysis, focused on severe CoV bronchiolitis, we grouped infants into two mutually exclusive CoV genus categories: alpha (NL63, 229E) and beta (HKU1, OC43). To derive metabolites that best discriminate the alpha and beta CoVs, we performed a sparse partial least-squares discriminate analysis (sPLS-DA) with Lasso penalization and cross-validation to minimize potential overfitting, using $\mathrm{R}$ mixOmics package. ${ }^{12}$ For the downstream analyses, we selected the top 30 metabolites that had a high variable importance. Next, we computed Spearman's correlations between viral genomic load (measured as the inverse cycle threshold) and intensity of these selected 30 discriminatory metabolites. We then tested for the association of CoV groups (alpha genus as the reference) with each metabolite by constructing multivariable linear regression models adjusting for four potential confounders (age, co-infection, CoV genomic load, and batch of metabolomics testing) and patient clustering within hospitals to account for within-hospital patient clustering. To detect biologically meaningful pathways, we also performed a pathway analysis (metabolite-set enrichment analysis) using MetaboAnalyst 4.0. To ensure that metabolomic signatures are not fully driven by respiratory syncytial virus (RSV) coinfections, we also repeated sPLS-DA model to compare solo RSV and CoV/ RSV infections. We also examined the association of CoV groups with four distinct nasopharyngeal microbiota profiles according to a clustering method as previously described. ${ }^{11}$ The data were analyzed using $\mathrm{R}$ version 3.6.1 (R Foundation, Vienna, Austria). The institutional review board at each of the participating hospitals approved the study. Informed consent was obtained from all parents or guardians.

\section{RESULTS}

Of 1016 infants enrolled in the MARC-35 cohort, CoV was detected in 65 infants. Of these, we excluded four infants who had both alpha and beta CoV detection and one infant who had insufficient sequence depth for the microbiome testing. The remaining 60 infants with severe bronchiolitis-31 alpha CoVs (NL63, 229E) and 29 beta CoVs (HKU1, OC43)-comprised the analytic cohort. Overall, the median age was 3 months, $65 \%$ were males, $75 \%$ were co-infected with RSV, and $22 \%$ underwent intensive care treatment. Between the two CoV groups, there were no significant differences in most patient characteristics or clinical outcomesexcept for the proportion of breastfed infants $(P=0.049)$-in this sample of infants with severe bronchiolitis (Table 1 ).

The nasopharyngeal metabolomic profiling identified a total of 285 metabolites from 76 sub-pathways within 7 super-pathways. Based on the sPLS-DA, the overall metabolome profiles in each of these two CoV groups clustered with a partial overlap (Fig. 1a). The sPLS-DA selected 30 discriminatory metabolites-primarily 
Table 1. Patient characteristics of infants with severe bronchiolitis, according to coronavirus genera.

\begin{tabular}{|c|c|c|c|}
\hline & $\begin{array}{l}\text { Alpha CoV (NL63, } \\
229 \mathrm{E})(n=31 ; 52 \%)\end{array}$ & $\begin{array}{l}\text { Beta CoV (HKU1, } \\
\text { OC43) }(n=29 ; 48 \%)\end{array}$ & $P$ value \\
\hline \multicolumn{4}{|l|}{ Characteristics } \\
\hline Age (month), median (IQR) & $3(2-5)$ & $3(2-5)$ & 0.71 \\
\hline Male sex & $13(42)$ & $8(28)$ & 0.37 \\
\hline Race/ethnicity & & & 0.06 \\
\hline Non-Hispanic white & $17(55)$ & $13(45)$ & \\
\hline Non-Hispanic black & $10(32)$ & $4(14)$ & \\
\hline Hispanic & $4(13)$ & $11(38)$ & \\
\hline Other or unknown & $0(0)$ & $1(3)$ & \\
\hline $\begin{array}{l}\text { Maternal smoking during } \\
\text { pregnancy }\end{array}$ & $6(19)$ & $3(10)$ & 0.50 \\
\hline C-section delivery & $7(23)$ & $4(14)$ & 0.59 \\
\hline Prematurity (32-37 weeks) & $6(19)$ & $5(17)$ & 0.99 \\
\hline History of eczema & $6(19)$ & $4(14)$ & 0.82 \\
\hline $\begin{array}{l}\text { Mostly breastfed for the first } \\
3 \text { month of age }\end{array}$ & $12(39)$ & $19(66)$ & 0.049 \\
\hline $\begin{array}{l}\text { Cigarette smoke exposure } \\
\text { at home }\end{array}$ & $4(13)$ & $5(17)$ & 0.91 \\
\hline $\begin{array}{l}\text { Corticosteroid use before the } \\
\text { index hospitalization }\end{array}$ & $3(10)$ & $1(3)$ & 0.66 \\
\hline $\begin{array}{l}\text { Lifetime history of systemic } \\
\text { antibiotic use }\end{array}$ & $8(26)$ & $11(38)$ & 0.47 \\
\hline \multicolumn{4}{|l|}{ Clinical presentation } \\
\hline $\begin{array}{l}\text { Weight at presentation (kg), } \\
\text { median (IQR) }\end{array}$ & $5.3(4.1-6.2)$ & $6.1(4.7-7.6)$ & 0.14 \\
\hline $\begin{array}{l}\text { Respiratory rate at presentation } \\
\text { (per minute), median (IQR) }\end{array}$ & $50(40-60)$ & $53(40-68)$ & 0.39 \\
\hline $\begin{array}{l}\text { Oxygen saturation at } \\
\text { presentation }\end{array}$ & & & 0.58 \\
\hline$<90 \%$ & $1(3)$ & $2(7)$ & \\
\hline $90-93 \%$ & $7(23)$ & $4(14)$ & \\
\hline$\geq 94 \%$ & $23(74)$ & $23(79)$ & \\
\hline $\begin{array}{l}\text { Serum free } 25 \text {-hydroxyvitamin D } \\
\text { (pg/mL), median (IQR) }\end{array}$ & $16.2(9.9-21.4)$ & $11.7(6.3-17.9)$ & 0.29 \\
\hline \multicolumn{4}{|l|}{ Detected pathogen } \\
\hline \multicolumn{4}{|l|}{ Coronavirus species } \\
\hline NL63 & $23(74)$ & $0(0)$ & - \\
\hline $229 \mathrm{E}$ & $9(29)$ & $0(0)$ & - \\
\hline HKU1 & $0(0)$ & $16(55)$ & - \\
\hline OC43 & $0(0)$ & $14(48)$ & - \\
\hline Any co-infection & $27(87)$ & $25(86)$ & 0.99 \\
\hline Respiratory syncytial virus & $21(68)$ & $24(83)$ & 0.30 \\
\hline Rhinovirus & $5(16)$ & $4(14)$ & 0.99 \\
\hline Other pathogen ${ }^{a}$ & $11(35)$ & $2(7)$ & 0.02 \\
\hline \multicolumn{4}{|l|}{ Acute clinical outcomes } \\
\hline Positive pressure ventilation ${ }^{\mathrm{b}}$ & $3(10)$ & $5(17)$ & 0.63 \\
\hline Intensive treatment ${ }^{c}$ & $7(23)$ & $6(21)$ & 0.99 \\
\hline $\begin{array}{l}\text { Hospital length of stay (day), } \\
\text { median (IQR) }\end{array}$ & $2(1-3)$ & $2(2-5)$ & 0.39 \\
\hline \multicolumn{4}{|l|}{ Chronic clinical outcomes } \\
\hline Recurrent wheezing by 3 years ${ }^{d}$ & $8(26)$ & $8(28)$ & 0.99 \\
\hline Asthma at 5 years $^{\mathrm{e}}$ & $4(13)$ & $9(31)$ & 0.17 \\
\hline
\end{tabular}

CoV coronavirus, $I Q R$ interquartile range.

Data are $n(\%)$ of infants unless otherwise indicated.

adenovirus, bocavirus, Bordetella pertussis, enterovirus, human metapneumovirus, influenza A or B virus, Mycoplasma pneumoniae, parainfluenza virus 1-3.

${ }^{b}$ Defined as use of invasive and/or non-invasive mechanical ventilation (e.g., continuous positive airway pressure ventilation).

${ }^{\mathrm{C} D e f i n e d}$ as use of positive pressure ventilation and/or intensive care unit admission.

${ }^{\mathrm{d}}$ Defined by parental report of at least two corticosteroid-requiring breathing problems in 6 months or at least four wheezing episodes in one year that last at least one day and affect sleep.

eDefined by physician-diagnosis of asthma at age 5 years, plus either asthma medication use (e.g., albuterol inhaler, inhaled corticosteroids, montelukast) or asthma-related symptoms in the preceding year. involved in lipid metabolism—such as lysoplasmalogen, lysophospholipid, and phospholipid metabolism (Fig. 1a). In the multivariable models adjusting for potential confounders (e.g., coinfection status), the between-virus differences remained significant for 11 metabolites $(P<0.05$; Table E2). For example, compared to the alpha $\mathrm{CoV}$ group, the beta $\mathrm{CoV}$ group had a significantly higher intensity of glycerol-phosphoethanolamines (GPEs), glycero-phosphocholines, and glycosyl-phosphatidylinositol. In contrast, the beta CoV group had a significantly lower intensity of phosphocholine. These differences between the qualitative virus status were consistent with the correlations between quantitative virus status (genomic RNA load) and metabolite intensity (Fig. 1b). Consistently, the metabolite-set enrichment analysis also identified 11 enriched pathways-e.g., sphingolipid, phosphatidylcholine, and phosphatidylethanolamine metabolism pathways ( $P<0.05$; Table E3). Additionally, the sensitivity analysis that compares solo RSV and CoV/RSV infections showed differences in correlations of genomic load with metabolite intensities, suggesting that RSV co-infection alone cannot explain the CoV-specific metabolomic signatures (Fig. E1). In the comparison of nasopharyngeal airway microbiota, compared to infants with alpha CoV, those with beta CoV bronchiolitis had a significantly higher proportion of Haemophilus-dominant profile (10\% vs. $31 \%, P=0.04$; Fig. E2 and Table E4).

\section{DISCUSSION}

In this multicenter prospective cohort of infants with severe CoV bronchiolitis, with comprehensive profiling of the nasopharyngeal airway metabolome and microbiome, we investigated potential differences between endemic alpha- and beta-CoVs. We found that, compared to alpha $\mathrm{CoV}$ infection, infants with beta $\mathrm{CoV}$ bronchiolitis had a distinct metabolomic signature that was primarily characterized by upregulated lipid mediators. Additionally, we also found between-CoV differences in the nasopharyngeal microbiota profile. To the best of our knowledge, this is the first investigation that has examined the relations of CoV genera with the metabolome and microbiota among children with acute respiratory infection.

Although bronchiolitis has been conventionally considered a single disease entity that has similar mechanisms and clinical characteristics, ${ }^{8}$ concordant with the current study, recent studies demonstrate important between-virus (RSV vs. rhinovirus, including different species) differences in the upper airway metabolome ${ }^{9,13}$ and microbiome ${ }^{14,15}$ profiles. The current study corroborates these prior reports, and extends them by demonstrating between-CoV differences in these molecular characteristics among infants with severe bronchiolitis. Our data showed that infants with beta CoV bronchiolitis had a higher intensity of lysoplasmalogens-e.g., 1-(1enyl-oleoyl)-GPE. These lipids are known to function as antiinflammatory mediators and have protective effects against oxidation of lung surfactant lipids ${ }^{16}$ a composite of phosphatidylcholine, phosphatidylinositol, and phosphatidylethanolamine which metabolism pathways were also differentially enriched in our data. Additionally, research has demonstrated a decreased level of plasmalogens in premature infants with bronchopulmonary dysplasia. ${ }^{17}$ We also found that phosphocholine-an essential substrate of sphingolipids-is a discriminatory metabolite. Sphingolipids are not only major structural component of cellular membranes but also lipid mediators that play important roles in immune response and inflammation. ${ }^{18}$ In addition to the between-CoV differences in the airway metabolome, we found that infants with beta-CoV bronchiolitis had a higher likelihood of Haemophilus-dominant microbiota profile, which has been associated with upregulated $\mathrm{T}_{\mathrm{H}} 2$ and $T_{H}$ 17-type inflammatory response in the airway. ${ }^{11}$ While the identification of exact mechanisms underlying the CoV-airway metabolites-microbiota relationship is beyond scope of the current study, these findings will help to advance research into the 
a

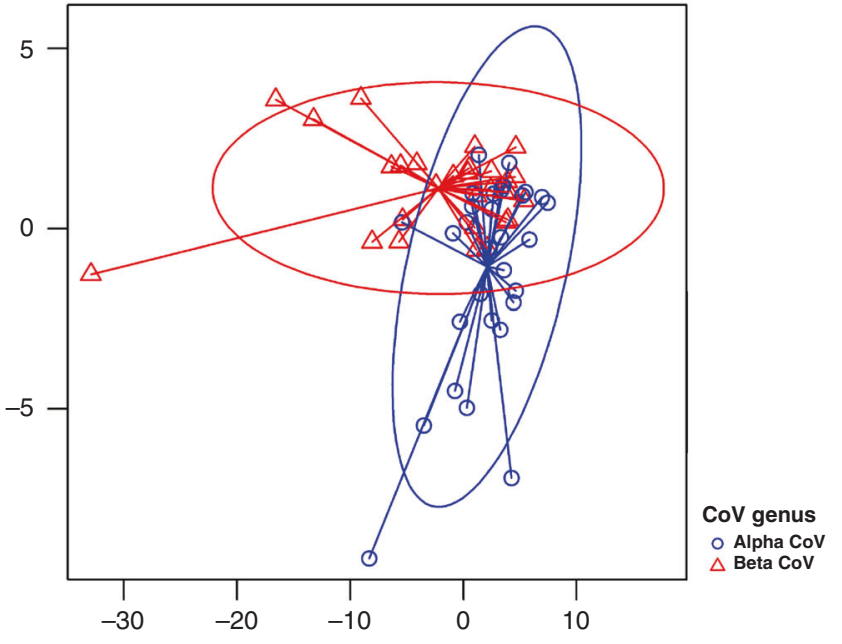

b

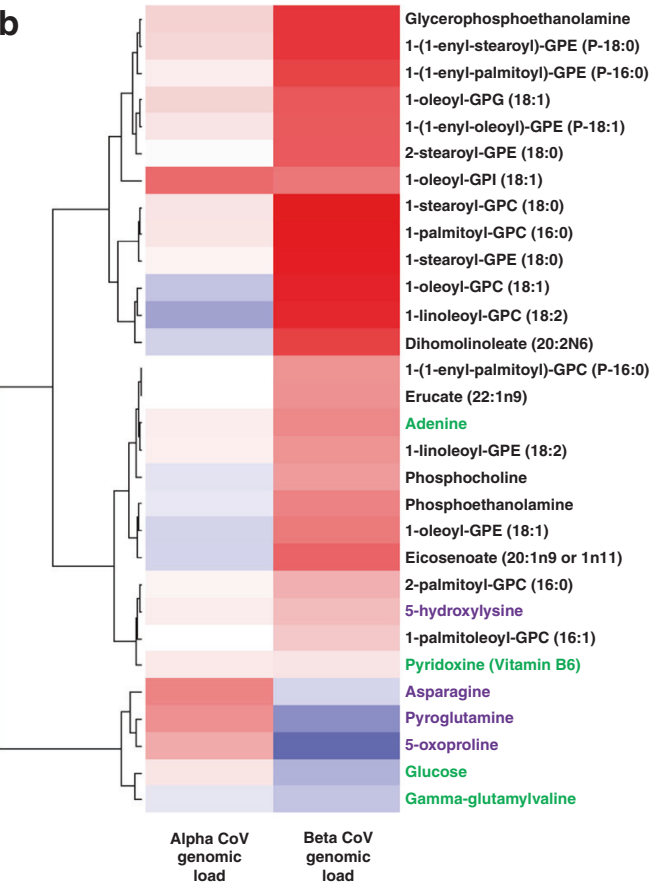

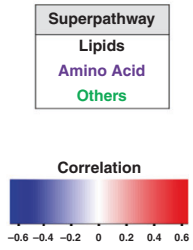

Fig. 1 Associations of coronavirus groups with nasopharyngeal airway metabolites in infants with severe bronchiolitis. a Sparse partial least-squares discriminant analysis (sPLS-DA) score plot of nasopharyngeal airway metabolome according to alpha (blue) vs. beta (red) CoV infection. Each dot represents the nasopharyngeal airway metabolome profile of a single infant, by plotting the component scores in the smaller subspace spanned by latent variables of sPLS-DA. The eclipses are $95 \%$ confidence intervals. The arrows start from the centroid of each virus group and end for each infant belonging to each group. b Heatmap of Spearman's correlations of the genomic load (measured as the inverse cycle threshold value) of alpha and beta CoVs with the 30 most discriminatory nasopharyngeal airway metabolites in the corresponding SPLS-DA model (Table E2). Clustering is based on Euclidean distance and Ward's minimum variance linkage algorithm. The color bar indicates the correlation coefficients-red color indicates a positive correlation while blue color indicates a negative correlation. Superpathway of each metabolite is color-coded for each function. CoV coronavirus.

integrated role of different CoVs, host immune response, and microbiome in the pathobiology of acute respiratory infection.

The underlying mechanisms of virus-metabolome-microbiota relationships in the bronchiolitis pathobiology merit further clarification. It is possible that the observed virus-metabolome-microbiota associations may be causal-that is, specific respiratory virus species induces distinct host response and metabolome signature, thereby leading to overgrowth of specific bacteria in the airway niche. Alternatively, unique metabolome and microbiota profiles in conjunction with airway immune response might have contributed to susceptibility to specific virus infection. These potential mechanisms are not mutually exclusive.

This study has several potential limitations. First, bronchiolitis involves inflammation of the lower airways in addition to the upper airways. The present study was based on the upper (nasopharyngeal) airway samples. Although research has shown that upper airway samples reliably represent the lung inflammation ${ }^{19}$ and microbiome ${ }^{20}$ profiles, further validation study using lower airways samples (e.g., bronchoalveolar lavage) is desirable. Second, the current study did not have "healthy controls." However, the study objective was not to compare the metabolome and microbiome between-CoV bronchiolitis and healthy controls, but to examine between-CoV differences in these molecular characteristics within infants with bronchiolitis. Third, because of the limited sample size, overfitting of data is possible, and this result may not ensure generalizability. Fourth, the majority of infants in this cohort were co-infected with RSV, while the sensitivity analysis demonstrated differences in the metabolomic signature between solo RSV and CoV/RSV infection groups. To specifically investigate the metabolome signature of solo CoV infections, the observed findings-albeit biologically-plausiblemerit additional investigations using a larger sample size of infants with solo CoV infection. Fifth, the inferences from this study using an untargeted and hypothesis-free metabolomics approach warrant further validation with the use of targeted approaches. Finally, although the cohort consisted of a racially/ethnically and geographically diverse sample, inferences should be cautiously generalized beyond US infants hospitalized for bronchiolitis.

In conclusion, these multicenter data from infants with severe bronchiolitis demonstrated that, despite the absence of apparent differences in clinical manifestations and a small sample size, alpha versus beta $\mathrm{CoV}$ differ in their underlying pathobiology. Compared to alpha CoV infection, infants with beta CoV infection had a distinct nasopharyngeal metabolome signature (with upregulated biologically active lipid mediators, even after adjusting for coinfections), as well as distinct nasopharyngeal microbiota profiles. Our data suggest that infection by beta CoVs (which also includes SARS-CoV-2) elicits unique downstream effects in the host airway, thereby contributing to distinct pathobiology. While causal inference remains to be elucidated, these findings should facilitate further investigations into the complex interplay between different CoVs and host airway response in children with acute respiratory infection.

\section{ACKNOWLEDGEMENTS}

We thank the MARC-35 study hospitals and research personnel for their ongoing dedication to bronchiolitis and asthma research (Table E1 in the Online Supplement), and Janice A. Espinola, MPH and Ashley F. Sullivan, MS, MPH (Massachusetts General Hospital, Boston, MA) for their many contributions to the MARC-35 study. We also thank Alkis Togias, MD, at the National Institutes of Health (Bethesda, MD) for helpful comments about the study results. The current study is supported by grants from the National Institutes of Health (Bethesda, MD): R01 Al-127507, R01 Al-134940, R01 Al137091, and UG3/UH3 OD-023253. The content of this manuscript is solely the responsibility of the authors and does not necessarily represent the official views of the National Institutes of Health. 


\section{AUTHOR CONTRIBUTIONS}

M.F. carried out the statistical analysis, drafted the initial manuscript, revised the manuscript, and approved the final manuscript as submitted. C.A.C. and K.H. conceptualized and designed the study, obtained the funding, reviewed and revised the manuscript, and approved the final manuscript as submitted. Y.R. and L.T. assisted in the statistical analysis, reviewed and revised the manuscript, and approved the final manuscript as submitted. R.J.F. conceptualized the study, reviewed and revised the manuscript, and approved the final manuscript as submitted. J.M.M. collected the study data, reviewed and revised the manuscript, and approved the final manuscript as submitted. P.A.P. generated virus data, reviewed and revised the manuscript, and approved the final manuscript as submitted.

\section{ADDITIONAL INFORMATION}

The online version of this article (https://doi.org/10.1038/s41390-020-01154-2) contains supplementary material, which is available to authorized users.

Competing interests: The authors declare no competing interests.

Publisher's note Springer Nature remains neutral with regard to jurisdictional claims in published maps and institutional affiliations.

Michimasa Fujiogi (D) ${ }^{1}$, Carlos A. Camargo $\mathrm{Jr}^{1}$, Yoshihiko Raita ${ }^{1}$, Laura Toivonen $^{1,2}$, Robert J. Freishtat ${ }^{3,4,5}$, Jonathan M. Mansbach ${ }^{6}$, Pedro A. Piedra ${ }^{7}$ and Kohei Hasegawa ${ }^{1}$

${ }^{1}$ Department of Emergency Medicine, Massachusetts General Hospital, Harvard Medical School, Boston, MA, USA; ${ }^{2}$ Department of Paediatrics and Adolescent Medicine, Turku University Hospital and University of Turku, Turku, Finland; ${ }^{3}$ Division of Emergency Medicine, Children's National Hospital, Washington, DC, USA; ${ }^{4}$ Department of Pediatrics, George Washington University School of Medicine and Health Sciences, Washington, DC, USA; ${ }^{5}$ Department of Genomics and Precision Medicine, George Washington University School of Medicine and Health Sciences, Washington, DC, USA; ${ }^{6}$ Department of Pediatrics, Boston Children's Hospital, Harvard Medical School, Boston, MA, USA and ${ }^{7}$ Departments of Molecular Virology and Microbiology and Pediatrics, Baylor College of Medicine, Houston, TX, USA

Correspondence: Michimasa Fujiogi (fujiogi-stm@umin.ac.jp)

\section{REFERENCES}

1. Raoult, D., Zumla, A., Locatelli, F., Ippolito, G. \& Kroemer, G. Coronavirus infections: epidemiological, clinical and immunological features and hypotheses. Cell Stress 4, 66-75 (2020).
2. Hasegawa, K. et al. Respiratory virus epidemiology among US infants with severe bronchiolitis: analysis of 2 multicenter, multiyear cohort studies. Pediatr. Infect. Dis. J. 38, E180-E183 (2019).

3. Killerby, M. E. et al. Human coronavirus circulation in the United States 2014-2017. J. Clin. Virol. 101, 52-56 (2018).

4. Chen, Y., Liu, Q. \& Guo, D. Emerging coronaviruses: genome structure, replication, and pathogenesis. J. Med. Virol. 92, 418-423 (2020).

5. Walsh, E. E., Shin, J. H. \& Falsey, A. R. Clinical impact of human coronaviruses $229 \mathrm{E}$ and OC43 infection in diverse adult populations. J. Infect. Dis. 208, 1634-1642 (2013).

6. Varghese, L. et al. Epidemiology and clinical features of human coronaviruses in the pediatric population. J. Pediatr. Infect. Dis. Soc. 7, 151-158 (2018).

7. Fielding, B. C. Human coronavirus NL63: a clinically important virus? Future Microbiol. 6, 153-159 (2011).

8. Ralston, S. L. et al. Clinical practice guideline: the diagnosis, management, and prevention of bronchiolitis. Pediatrics 134, e1474-e1502 (2014).

9. Stewart, C. J. et al. Respiratory syncytial virus and rhinovirus bronchiolitis are associated with distinct metabolic pathways. J. Infect. Dis. 217, 1160-1169 (2018).

10. Fujiogi, M. et al. Association of rhinovirus species with nasopharyngeal metabolome in bronchiolitisinfants: a multicenterstudy. Allergy https://doi.org/ 10.1111/all.14326 (2020).

11. Hasegawa, K. et al. Association of nasopharyngeal microbiota profiles with bronchiolitis severity in infants hospitalised for bronchiolitis. Eur. Respir. J. 48, 1329-1339 (2016).

12. Rohart, F., Gautier, B., Singh, A. \& Le Cao, K.-A. mixOmics: an R package for'omics feature selection and multiple data integration. PLoS Comput. Biol. 13, e1005752 (2017).

13. Fujiogi, M. et al. In infants with severe bronchiolitis: dual-transcriptomic profiling of nasopharyngeal microbiome and host response. Pediatr. Res. https://doi.org/ 10.1038/s41390-019-0742-e1005758 (2020).

14. Toivonen, L. et al. Association between rhinovirus species and nasopharyngeal microbiota in infants with severe bronchiolitis. J. Allergy Clin. Immunol. 143, 1925-1928.e7 (2019).

15. Rosas-Salazar, C. et al. Differences in the nasopharyngeal microbiome during acute respiratory tract infection with human rhinovirus and respiratory syncytial virus in infancy. J. Infect. Dis. 214, 1924-1928 (2016).

16. Braverman, N. E. \& Moser, A. B. Functions of plasmalogen lipids in health and disease. Biochim. Biophys. Acta 1822, 1442-1452 (2012).

17. Rüdiger, M., Von Baehr, A., Haupt, R., Wauer, R. R. \& Rüstow, B. Preterm infants with high polyunsaturated fatty acid and plasmalogen content in tracheal aspirates develop bronchopulmonary dysplasia less often. Crit. Care Med. 28, 1572-1577 (2000).

18. Sturgill, J. L. Sphingolipids and their enigmatic role in asthma. Adv. Biol. Regul. 70, 74-81 (2018).

19. Poole, A. et al. Dissecting childhood asthma with nasal transcriptomics distinguishes subphenotypes of disease. J. Allergy Clin. Immunol. 133, 670-678.e12 (2014).

20. Marsh, R. L. et al. The microbiota in bronchoalveolar lavage from young children with chronic lung disease includes taxa present in both the oropharynx and nasopharynx. Microbiome 4, 1-18 (2016). 